\title{
Icariin protects H9c2 cardiomyocytes from lipopolysaccharide-induced injury via inhibition of the reactive oxygen species-dependent c-Jun $\mathrm{N}$-terminal kinases/nuclear factor- $\mathrm{kB}$ pathway
}

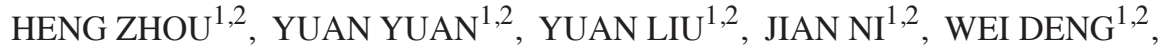 \\ ZHOU-YAN BIAN ${ }^{1,2}$, JIA DAI $^{1,2}$ and QI-ZHU TANG ${ }^{1,2}$ \\ ${ }^{1}$ Department of Cardiology, Renmin Hospital of Wuhan University; \\ ${ }^{2}$ Cardiovascular Research Institute of Wuhan University, Wuhan, Hubei 430060, P.R. China
}

Received February 7, 2014; Accepted December 9, 2014

DOI: $10.3892 / \mathrm{mmr} .2015 .3289$

\begin{abstract}
The inflammatory response is involved in the pathogenesis of the most common forms of heart disease. Icariin has a number of pharmacological actions, including anti-inflammatory, anti-oxidative and anti-apoptotic effects. However, the role of icariin in cardiac inflammation has remained elusive. In the present study, H9c2 rat cardiomyocytes were stimulated by lipopolysaccharide (LPS) and treated with icariin. The results showed that icariin significantly reduced the increase in the mRNA expression of tumor necrosis factor $\alpha$, interleukin (IL)-1 $\beta$ and IL- 6 that occurred in response to LPS. Furthermore, icariin regulated the expression of B-cell lymphoma 2 and B-cell lymphoma 2-associated X, and rescued H9c2 cells from apoptosis. Incubation with 2',7'-dichlorofluorescein diacetate demonstrated that icariin inhibited the production of intracellular reactive oxygen species (ROS). In addition, the phosphorylation of c-Jun N-terminal kinases (JNK), the degradation of inhibitor of $\kappa \mathrm{B}$ and the nuclear translocation of nuclear factor- $\kappa \mathrm{B}(\mathrm{NF}-\kappa \mathrm{B}) \mathrm{p} 65$ in LPS-treated H9c2 cells were blocked by icariin treatment. These results suggested that icariin prevented cardiomyocytes from inflammatory response and apoptosis, and that this effect may be mediated by inhibition of the ROS-dependent JNK/NF- $\mathrm{B}$ pathway.
\end{abstract}

\section{Introduction}

The inflammatory response is involved in the pathogenesis of the most common forms of heart disease, including

Correspondence to: Professor Qi-Zhu Tang, Department of Cardiology, Renmin Hospital of Wuhan University, 238 Jiefang Road, Wuhan, Hubei 430060, P.R. China

E-mail: qztang@whu.edu.cn

Key words: epimedium, inflammation, apoptosis, c-Jun N-terminal kinase, nuclear factor- $\kappa \mathrm{B}$ myocarditis, cardiomyopathy, myocardial infarction and heart failure (1). Although the initial inflammatory reaction is a protective response to stressors, including infection or tissue injury, prolonged inflammation leads to additional cardiac injury and cardiomyocyte loss (2). Of note, cardiomyocytes themselves are a significant source of pro-inflammatory cytokines, including tumor necrosis factor $\alpha(\mathrm{TNF}-\alpha)$, interleukin (IL)-1 $\beta$ and IL-6 (3). Toll-like receptor 4 (TLR4), an innate immune receptor expressed on immune cells and cardiomyocytes, is critical in the activation of the nuclear factor- $\kappa \mathrm{B}$ $(\mathrm{NF}-\kappa \mathrm{B})$ pathway and the induction of inflammatory cytokines within cardiomyocytes (4). TLR4 signaling is involved in numerous cardiovascular diseases, including myocardial ischemia, ischemia/reperfusion, cardiac hypertrophy, cardiomyopathy and heart failure (5-9). Therefore, pharmacological interventions which disrupt the TLR4-induced inflammatory response in cardiomyocytes may be a promising approach for the treatment of certain cardiovascular diseases.

Icariin (ICA; $\mathrm{C}_{33} \mathrm{H}_{40} \mathrm{O}_{15} ; \mathrm{MW}, 676.66$ ), a prenylated flavonol glycoside, is the major active component isolated from plants of the Epimedium family, which are used in Traditional Chinese Medicine for the treatment of rheumatism, osteoporosis and hypogonadism $(10,11)$. Icariin has an anti-inflammatory effect in a number of tissues and cells, and a range of pharmacological properties of icariin have been revealed, including immunoregulation, regulation of oxidative stress, anti-apoptotic effects and stimulation of angiogenesis (10,12-14). Recently, Song et al (13) showed that icariin attenuated cardiac remodeling in rats with congestive heart failure through inhibition of matrix metalloproteinase (MMP) activity and protection of cardiomyocytes from apoptosis. This suggests that icariin has a cardioprotective role. However, it has yet to be elucidated whether icariin inhibits the inflammatory response and subsequent injury in stimulated cardiomyocytes.

Lipopolysaccharide (LPS), a component of the outer layer of the gram-negative bacterial cell wall, is an agonist of TLR4 and has been shown to induce cardiomyocyte dysfunction by activating TLR4 and promoting the production of inflammatory mediators (4). In the present study, LPS was used to induce 
inflammatory injury in $\mathrm{H} 9 \mathrm{c} 2$ rat cardiomyocytes treated with icariin, and the effects on cell viability, apoptosis and the production of inflammatory mediators were investigated. In addition, the possible mechanisms underlying the effects of icariin on these processes were examined.

\section{Materials and methods}

Reagents. Icariin ( $\geq 94 \%$ purity as determined by high performance liquid chromatography analysis), LPS and 2',7'-dichlorofluorescin diacetate (DCFH-DA) were obtained from Sigma-Aldrich (St. Louis, MO, USA). Dulbecco's modified Eagle's medium (DMEM)/F12, fetal bovine serum (FBS), trypsin, penicillin and streptomycin were obtained from Gibco-BRL (Invitrogen Life Technologies, Carlsbad, CA, USA). TRIzol ${ }^{\circledR}$ was obtained from Invitrogen Life Technologies. Transcriptor First Strand cDNA Synthesis kit and LightCycler ${ }^{\circledR} 480$ SYBR Green I Master mix were obtained from Roche Diagnostics (Basel, Switzerland). ApopTag ${ }^{\circledR}$ Plus Fluorescein In Situ Apoptosis Detection kit was obtained from EMD Millipore (Billerica, MA, USA). The following primary rabbit $\mathrm{IgG}$ antibodies were obtained from Cell Signaling Technology, Inc. (Danvers, MA, USA): GAPDH, P-JNK, T-JNK, P-IкB, T-IкB, P-P65 and T-P65 (1:1000; incubation overnight with gentle shaking at $4^{\circ} \mathrm{C}$ ). IRDye $800 \mathrm{CW}$ conjugated secondary antibodies were obtained from LI-COR Biosciences (Lincoln, NE, USA).

H9c2 cardiomyocyte culture. The H9c2 embryonic rat heart-derived cell line was obtained from the Cell Bank of the Chinese Academy of Sciences (Shanghai, China). Icariin was dissolved in dimethyl sulfoxide (DMSO; Sigma-Aldrich) at a concentration of $10 \mathrm{mmol} / \mathrm{l}$ for storage at $-20^{\circ} \mathrm{C}$. Cells were cultured in DMEM/F12 1:1 medium supplemented with 10\% FBS, penicillin $(100 \mathrm{U} / \mathrm{ml})$ and streptomycin $(100 \mathrm{mg} / \mathrm{ml})$ in a humidified incubator with an atmosphere of $5 \% \mathrm{CO}_{2}$ at $37^{\circ} \mathrm{C}$. Cells were seeded at a density of $1 \times 10^{6}$ per well onto six-well culture plates for, mRNA extraction; $5 \times 10^{5}$ per well onto six-well culture plates, for terminal deoxynucleotidyl transferase-mediated dUTP nick end-labeling (TUNEL) analysis; $5 \times 10^{3}$ cells per well in 96-well plates for reactive oxygen species (ROS) detection; and $1 \times 10^{7}$ per well onto culture dishes $(100 \mathrm{~mm})$, for protein extraction. Cells were cultured in serum-free medium for $24 \mathrm{~h}$ and pre-treated with icariin for $12 \mathrm{~h}$ prior to LPS stimulation.

Cell viability. Cell viability was investigated using a Cell Counting kit-8 (CCK-8; Sigma-Aldrich) assay. Following icariin treatment for $12 \mathrm{~h}, 10 \mu \mathrm{l} \mathrm{CCK}-8$ solution was added to each well of the 96-well plate prior to a further $4 \mathrm{~h}$ of incubation. Absorbance was measured at $450 \mathrm{~nm}$ using a microplate reader (Synergy ${ }^{\mathrm{TM}}$ HT, BioTek Instruments, Inc., Winooski, VT, USA). The percentage of cell viability was calculated according to the formula: Cell viability $(\%)=$ optical density (OD) of treatment group/OD of control group x100.

Reverse transcription-quantitative polymerase chain reaction $(R T-q P C R)$. RT-qPCR was used to detect the mRNA expression levels of inflammatory markers, including TNF- $\alpha$, IL-1 $\beta$ and IL-6, as described previously (15). Following $1 \mathrm{~h}$ pre-treatment with icariin, H9c2 cells were incubated with LPS for $12 \mathrm{~h}$ prior to the extraction of total RNA using TRIzol, and their yields and purities were spectrophotometrically estimated using the A260/A280 and A230/260 ratios via a SmartSpec Plus Spectrophotometer (Bio-Rad Laboratories, Hercules, CA, USA). RNA ( $2 \mu \mathrm{g}$ from each sample) was reverse-transcribed into cDNA using oligo (DT) primers (Sangon Biotech Co., Ltd., Shanghai, China) and the Transcriptor First Strand cDNA Synthesis kit. The primer sequences used were as follows: GAPDH, F 5'-GACATGCCG CCTGGAGAAAC-3' and R 5'-AGCCCAGGATGCCCTTTA GT-3'; TNF- $\alpha$, F 5'-AGCATGATCCGAGATGTGGAA-3' and R 5'-TAGACAGAAGAGCGTGGTGGC-3'; IL-1 $\beta$, F 5'-GGG ATGATGACGACCTGCTAG-3' and R 5'-ACCACTTGTTGG CTTATGTTCTG-3'; IL-6, F 5'-GTTGCCTTCTTGGGACTG ATG-3' and R 5'-ATACTGGTCTGTTGTGGGTGGT-3'; Bax, F 5'-AAACTGGTGCTCAAGGCCCT-3' and R 5'-AGCAGC CGCTCACGGAG-3'; Bcl-2, F 5'-CCGGGAGAACAGGGT ATGATAA-3' and R 5'-CCCACTCGTAGCCCCTCTG-3'. PCR amplifications were quantified using the LightCycler 480 SYBR Green I Master mix. GAPDH was used as the internal control. The PCR cycling conditions were as follows: Initial activation at $95^{\circ} \mathrm{C}$ for $10 \mathrm{~min}$, followed by 40 cycles of $95^{\circ} \mathrm{C}$ for $15 \mathrm{sec}$ and $60^{\circ} \mathrm{C}$ for $1 \mathrm{~min}$.

TUNEL staining. A TUNEL assay was performed to label apoptotic nuclei according to the manufacturer's instructions (ApopTag Plus Fluorescein In Situ Apoptosis Detection kit) (16). Briefly, following $1 \mathrm{~h}$ pre-treatment with icariin, cells were incubated with LPS for $12 \mathrm{~h}$ and subsequently fixed on coverslips in $1 \%$ paraformaldehyde in phosphate-buffered saline (both from Sinopharm Chemical Reagent Co., Ltd., Shanghai, China), stained with TUNEL reagents (EMD Millipore) and DAPI (Invitrogen Life Technologies) and observed under a microscope (BX51; Olympus Corp., Tokyo, Japan). The index of cell apoptosis was calculated as the percentage of apoptotic nuclei/total number of nuclei.

ROS detection. Intracellular ROS generation was determined by 2 ', 7 '-DCFH-DA which is oxidized to fluorescent DCF by ROS. Following $1 \mathrm{~h}$ pre-treatment with icariin, cells were incubated with LPS for 30, 60 or 120 min. Subsequently, H9c2 cells were washed twice and incubated with $5 \mu \mathrm{M}$ DCFH-DA solution in serum-free medium at $37^{\circ} \mathrm{C}$ for $30 \mathrm{~min}$ in the dark. Data were then collected using a fluorescence reader (Synergy HT, BioTek Instruments, Inc.) at an excitation/emission wavelength of 485/530 nm. A fluorescence microscope (BX51; Olympus Corp.) was also used to evaluate the DCF fluorescence of cells on coverslips.

Western blot analysis. Western blotting was performed as described previously (17). Following $1 \mathrm{~h}$ pre-treatment with icariin, cells were incubated with LPS for $2 \mathrm{~h}$. Cells were subsequently lysed in radioimmunoprecipitation assay lysis buffer (Guge Biological Technology Co., Wuhan, China), and the protein concentration was measured using a bicinchoninic acid protein assay kit (Thermo Fisher Scientific, Waltham, MA, USA) by a microplate reader (Synergy HT, BioTek Instruments, Inc.). Cell lysates $(50 \mu \mathrm{g})$ were electrophoresed by $10 \%$ SDS-PAGE, transferred onto Immobilon-FL transfer 


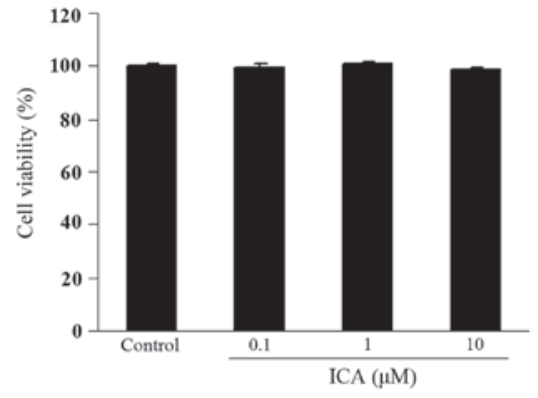

Figure 1. Effect of ICA on cell viability measured by Cell Counting kit- 8 assay. Treatment with ICA $(0.1,1$ or $10 \mu \mathrm{M})$ for $12 \mathrm{~h}$ did not cause a significant change in cell viability compared with that of the control group. ICA, icariin.

membranes (EMD Millipore), blocked with 5\% non-fat milk and incubated with specific primary antibodies overnight at $4^{\circ} \mathrm{C}$ prior to incubation with IRDye $800 \mathrm{CW}$-conjugated secondary antibodies. The blots were scanned using a two-color infrared imaging system (Odyssey, LI-COR Biosciences).

Statistical analysis. Values are presented as the mean \pm standard error of the mean. Differences among the groups were determined by two-way analysis of variance followed by a post hoc Tukey test. Comparisons between two groups were performed using the unpaired Student's t-test. $\mathrm{P}<0.05$ was considered to indicate a statistically significantly difference.

\section{Results}

Effect of icariin on cell viability. The potential cytotoxicity of icarrin was examined using a CCK-8 assay. H9c2 cells were incubated with varying concentrations of icariin (0.1, 1 and $10 \mu \mathrm{M}$ ) for $12 \mathrm{~h}$. Cell viability in icariin-treated cells was not significantly different compared with that of control cells, indicating that icariin $(0.1,1$ and $10 \mu \mathrm{M})$ did not cause cytotoxicity in H9c2 cells (Fig. 1).

Icariin inhibits the expression of inflammatory genes induced by LOS in $\mathrm{H} 9 \mathrm{c} 2$ cells. The effect of icariin at different concentrations $(0.1-10 \mu \mathrm{M})$ on the induction of TNF- $\alpha$, IL-1 $\beta$ and IL-6 in response to LPS was measured. Stimulation with LPS for $12 \mathrm{~h}$ significantly increased the mRNA levels of TNF- $\alpha$, IL-1 $\beta$ and IL-6 in H9c2 cells. In turn, icariin treatment significantly attenuated this increase in a concentration-dependent manner (Fig. 2).

Icariin attenuates LPS-induced apoptosis in H9c2 cells. To investigate the possible protective role of icariin in moderating LPS-induced apoptosis of H9c2 cells, TUNEL staining was used to identify apoptotic nuclei. A significant increase in the number of TUNEL-positive nuclei was observed in cells incubated with LPS, and icariin treatment markedly reduced this LPS-induced cell apoptosis (Fig. 3A and B). In addition, icariin decreased the levels of expression of B-cell-lymphoma (Bcl-2)-associated X (Bax) mRNA, while increasing the Bcl-2 mRNA expression levels in H9c2 cells following LPS stimulation (Fig. 3C). This mechanism may in part mediate the anti-apoptotic effect of icariin.
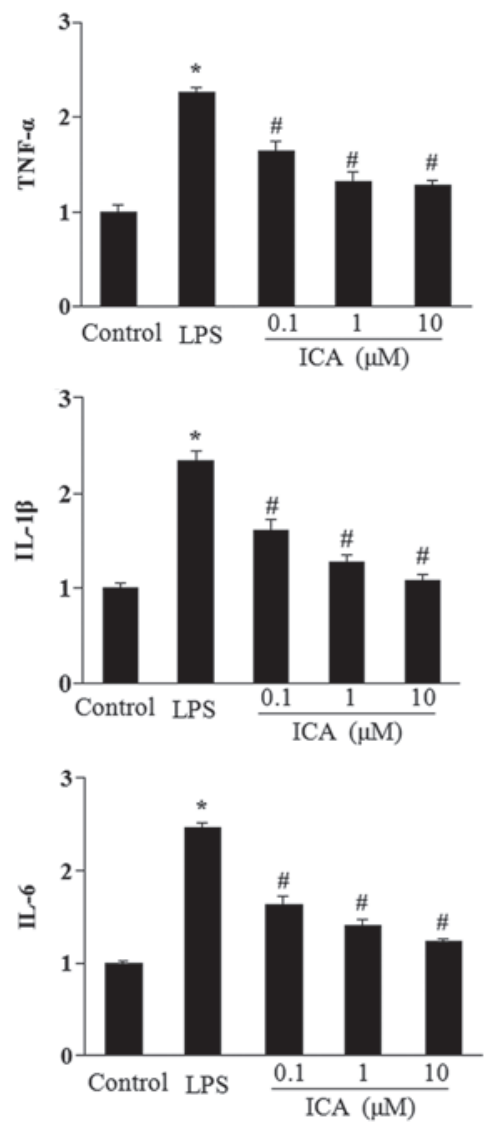

Figure 2. Effect of ICA on the mRNA expression of inflammatory mediators. ICA decreased LPS $(1 \mu \mathrm{g} / \mathrm{ml})$-induced expression of TNF- $\alpha$, IL-1 $1 \beta$ and IL-6 in a concentration- dependent manner. ${ }^{*} \mathrm{P}<0.05$, compared with control and ${ }^{\#} \mathrm{P}<0.05$, compared with cells treated with LPS. LPS, lipopolysaccharide; ICA, icariin; JNK, c-Jun N-terminal kinase; TNF- $\alpha$, tumor necrosis factor $\alpha$; IL, interleukin

Icariin decreases LPS-induced production of ROS. Cells incubated with DCFH-DA exhibited increased intensity of fluorescence when treated with LPS, indicating that LPS induced ROS production in a time-dependent manner (Fig. 4A). Icariin treatment significantly reduced LPS-induced ROS production at the time-points indicated in Fig. 4A. In addition, microscopic examination of DCF-derived fluorescence also suggested that icariin inhibited the accumulation of intracellular ROS in LPS-treated cells, which was in accordance with the results obtained with the fluorescence reader (Fig. 4B).

Icariin reduces the activation of $c$-Jun $N$-terminal kinase $(J N K)$ and $N F-\kappa B$ in response to LPS. To further investigate the mechanisms underlying the anti-inflammatory and anti-apoptotic effects of icariin on LPS-treated H9c2 cells, western blot analysis was used to detect the activation of JNK and NF- $\mathrm{\kappa B}$, which are key mediators in the cardiac inflammatory response. The results showed that the phosphorylated levels of JNK were significantly elevated by LPS, and that icariin treatment significantly inhibited this LPS-induced phosphorylation of JNK (Fig. 5A and B). Furthermore, icariin reduced the phosphorylation and degradation of IкB in $\mathrm{H} 9 \mathrm{c} 2$ cells that occurred in response to LPS administration, and subsequently decreased the nuclear translocation and phosphorylated level of NF-kB p65 (Fig. 5C-E). 
A
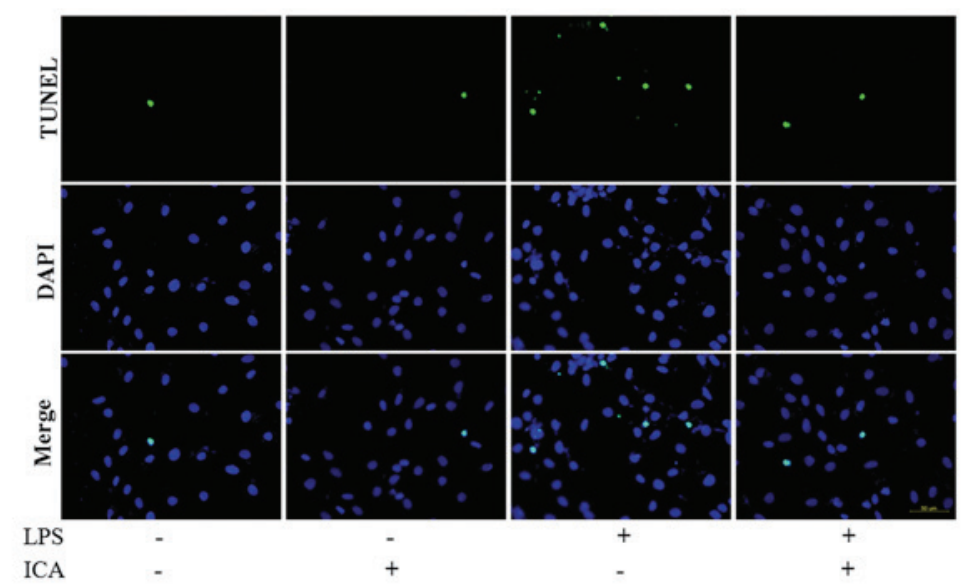

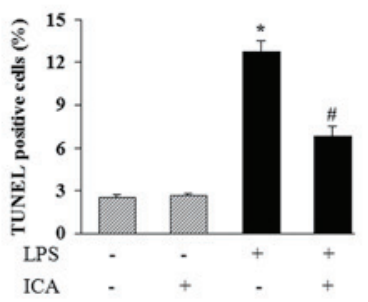

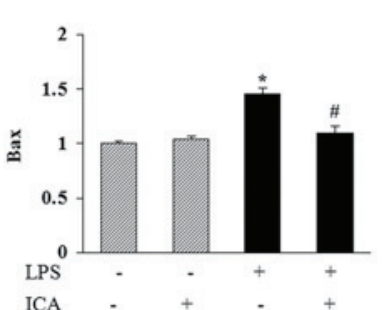

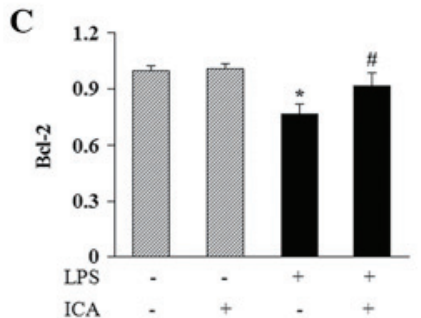

Figure 3. Effect of ICA on apoptosis. (A) and (B) Effect of icariin $(10 \mu \mathrm{M})$ on cell apoptosis following stimulation with LPS $(1 \mu \mathrm{g} / \mathrm{ml})$ for $12 \mathrm{~h}$ measured by TUNEL assay. (A) Representative images (magnification, $\mathrm{x} 400$ ) stained with TUNEL reagent and DAPI. (B) Quantitative results. (C) Effect of ICA (10 $\mu \mathrm{M})$ on mRNA levels of Bax and Bcl-2 in response to LPS. "P<0.05, compared with control cells and ${ }^{\#} \mathrm{P}<0.05$, compared with cells treated with LPS. LPS, lipopolysaccharide; ICA, icariin; TUNEL, terminal deoxynucleotide transferase-mediated dUTP nick end-labeling; Bcl-2, B-cell lymphoma 2; Bax, Bcl-2-associated x.

A

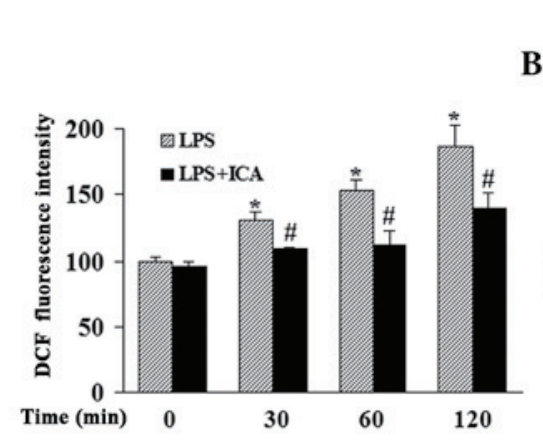

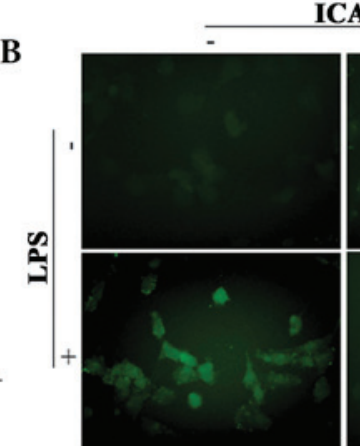

ICA
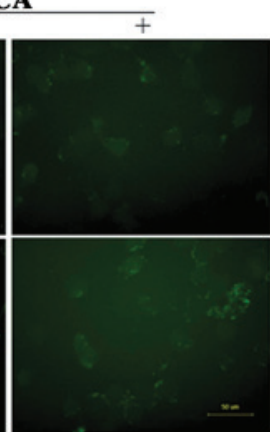

Figure 4. Effect of ICA on ROS production. (A) Effect of ICA $(10 \mu \mathrm{M})$ on LPS-induced ROS production at the indicated time-points, detected by a fluorescence reader. (B) Effect of ICA $(10 \mu \mathrm{M})$ on DCF-derived fluorescence in LPS-treated cells, detected using a fluorescence microscope (magnification, $\mathrm{x} 400)$. "P<0.05, compared with cells at the $0 \mathrm{~h}$ time-point and ${ }^{\mathrm{H}} \mathrm{P}<0.05$, compared with cells treated with LPS at the same time-point. ICA, icariin; ROS, reactive oxygen species; LPS, lipopolysaccharide; DCF, 2',7'-dichlorofluorescein.

\section{Discussion}

The present study demonstrated a novel role of icariin, a prenylated flavonol glycoside isolated from plants of the Epimedium family, in the protection of cardiomyocytes from the LPS-induced inflammatory response. Icariin also attenuated LPS-induced apoptosis in cardiomyocytes in association with the downregulation of Bax and the upregulation of Bcl-2. The protective effect of icariin on LPS-stimulated cardiomyocytes may be mediated by the alleviation of ROS production and inhibition of JNK activation and the NF- $\mathrm{\kappa B}$ signaling cascade.

A previous study showed that the inflammatory response induced by LPS in cardiomyocytes includes the initial induction of ROS, which leads to the activation of intracellular signaling pathways and transcription factors, and subsequently induces the production of inflammatory mediators, including TNF- $\alpha$, IL-1 $\beta$ and IL-6, and the apoptotic response $(18,19)$. These pro-inflammatory cytokines are involved in the depression of cardiac function and the progression from cardiac injury to failure (20). Therefore, blocking inflammatory signaling may produce beneficial effects in the dysfunctional heart (21).

Epimedium has been used in Traditional Chinese Medicine for the treatment of autoimmune disorders, such as rheumatism, for thousands of years, and its major active component, icariin, has an anti-inflammatory effect in certain tissues and cells $(10,11)$. Xu et al (10) showed that pretreatment with icariin decreased the expression of TNF- $\alpha$, IL- 6 , cycloxygenase- 2 
A

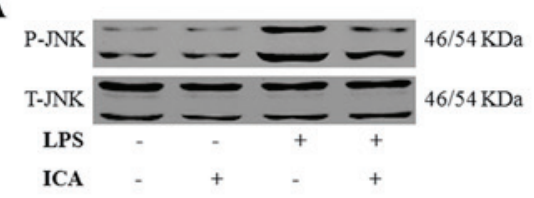

B

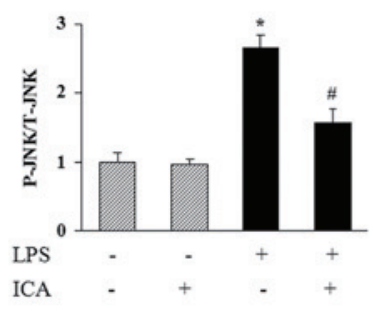

C
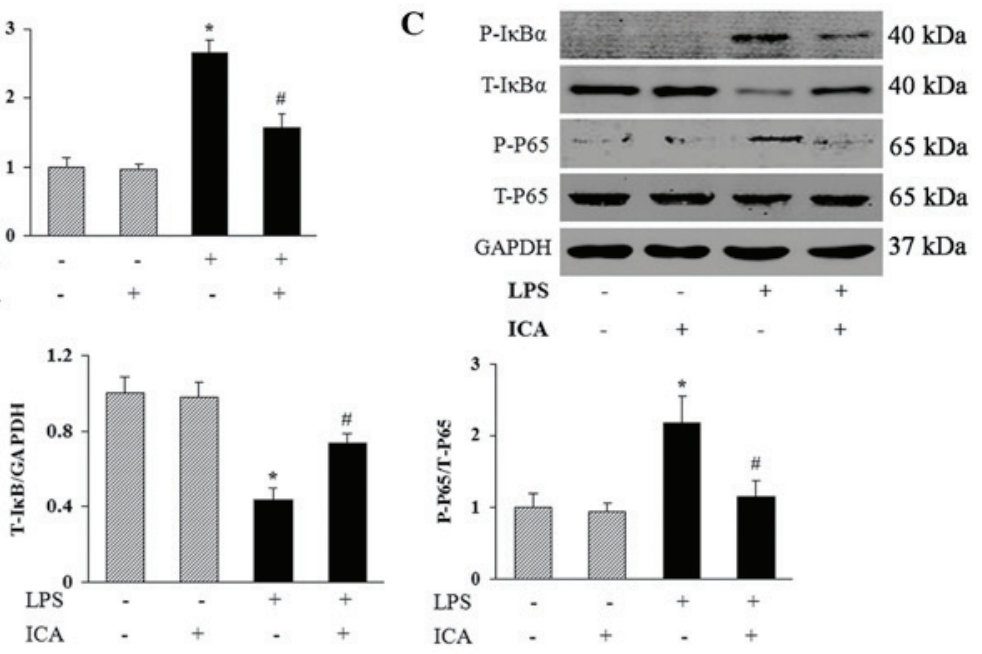

ICA

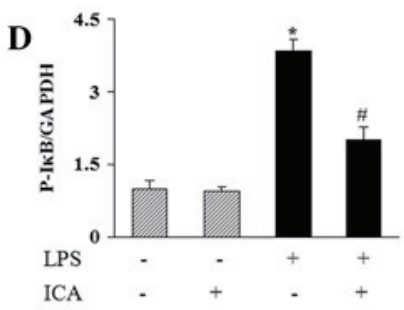

$\mathbf{E}$
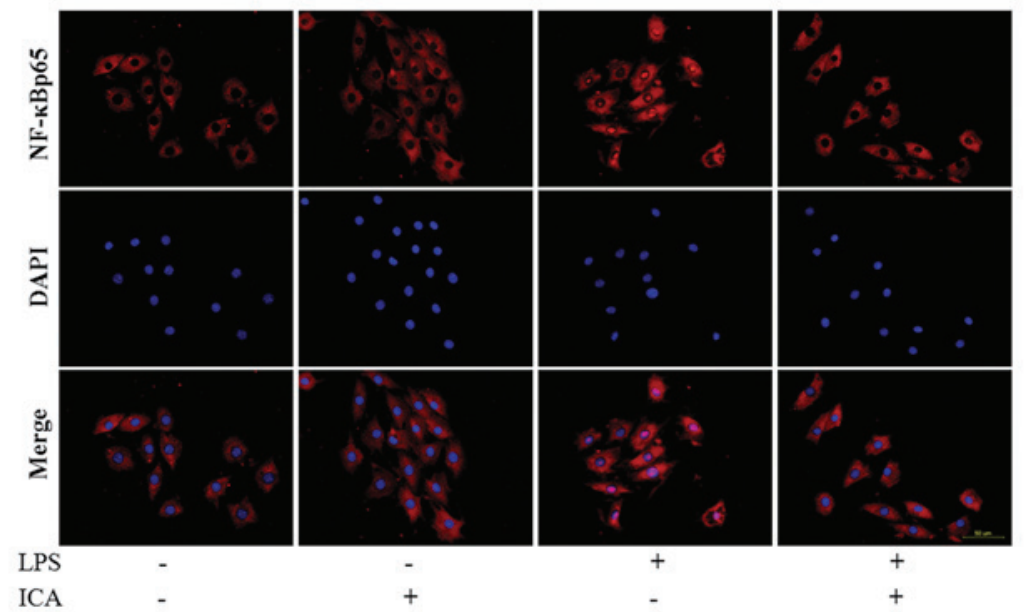

Figure 5. Effect of ICA on the activation of the JNK and NF-кB pathways. (A) and (B) Effect of ICA on the phosphorylated levels of JNK in response to LPS $(1 \mu \mathrm{g} / \mathrm{ml}$ for $2 \mathrm{~h})$. (C) and (D) Effect of ICA on the phosphorylation and degradation of IкB in H9c2 cells in response to LPS, and on the phosphorylated level of NF-кB p65. (A) and (C) Representative western blots. (B) and (D) Quantitative results. (E) Effect of ICA on the LPS-induced nuclear translocation of NF-kB p65 (magnification, $\mathrm{x} 400$ ). " $\mathrm{P}<0.05$, compared with control cells and ${ }^{\#} \mathrm{P}<0.05$, compared with cells treated with LPS. ICA, icariin; JNK, c-Jun

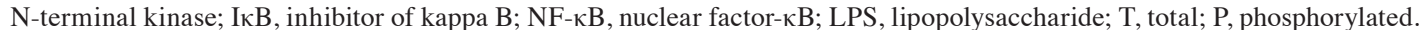

and prostaglandin E2 in the lungs of LPS-challenged mice. In addition, Zeng et al (22) demonstrated that icariin inhibited the release of TNF- $\alpha$, IL- $1 \beta$ and IL- 6 in cultured microglia that had been treated with LPS. The present study showed that icariin downregulated the expression of TNF- $\alpha$, IL-1 $\beta$ and IL- 6 in LPS-stimulated $\mathrm{H} 9 \mathrm{c} 2$ cells, indicating an anti-inflammatory effect of icariin in cardiomyocytes.

Inflammatory mediators may cause cardiac cytotoxicity and lead to cardiomyocyte loss through the induction of apoptotic pathways, thereby promoting cardiac dysfunction (23). Cardiomyocyte apoptosis is important in a number of cardiovascular diseases, including cardiac hypertrophy, heart failure, diabetic cardiomyopathy, ischemia/reperfusion injury, atherosclerosis and sepsis-associated cardiac dysfunction (19). The present study showed that icariin attenuated LPS-induced cardiomyocyte apoptosis, indicating a potential therapeutic role of LPS in heart disease. The balance between the regulation of pro-apoptotic (for example, Bax) and anti-apoptotic (for example, Bcl-2) proteins determines whether cells undergo apoptosis or survive. Icariin treatment downregulated the expression of Bax, while upregulating that of Bcl-2, in LPS-stimulated H9c2 cells. These changes may contribute to the anti-apoptotic effect of icariin.

As the results demonstrated that icariin attenuated the LPS-induced inflammatory response and apoptosis in H9c2 cells, the mechanisms underlying these beneficial effect were further investigated. In response to LPS, ROS generation is known to be markedly elevated, and is involved in the activation of signaling pathways and the production of inflammatory mediators (18). Previous studies have shown that icariin protects human umbilical vein endothelial cells from $\mathrm{H}_{2} \mathrm{O}_{2}$-induced apoptosis (24) and inhibits ROS production in LPS-treated microglia (22). These finding indicated that icariin has anti-oxidative effects. Studies have demonstrated that ROS production contributes to the LPS-induced activation of JNK, which belongs to the mitogen-activated protein kinase family and is involved in the induction of the inflammatory response and apoptosis $(25,26)$. The present study showed that icariin decreased ROS production and blocked the phosphorylation of JNK in LPS-treated H9c2 cells. The NF- $\kappa \mathrm{B} / \mathrm{Rel}$ 
family of transcription factors are key molecules that participate in the regulation of the inflammatory response, as well as certain other cellular processes, including cell growth, proliferation and apoptosis $(27,28)$. Inactive NF- $\kappa \mathrm{B}$ dimers (classically p65/p50) bind to cytosolic inhibitory proteins, I $\kappa$ Bs. Following stimulation of the $\mathrm{NF}-\kappa \mathrm{B}$ pathway, I $\kappa \mathrm{Bs}$ are phosphorylated, causing their ubiquitination and degradation, and the subsequent release and activation of NF- $\mathrm{B}$ (29). It has been reported that the $\mathrm{NF}-\kappa \mathrm{B}$ pathway may serve as a target of JNK (25). The present study suggested that icariin treatment inhibited the phosphorylation and degradation of $\mathrm{I} \kappa \mathrm{Bs}$ and blocked the subsequent nuclear translocation and phosphorylation of NF- $\mathrm{NB}$ p65 in response to LPS. This indicated that icariin may inhibit the NF- $\mathrm{B}$ pathway in association with the downregulation of ROS production and JNK activation.

In conclusion, the present study demonstrated for the first time, to the best of our knowledge, the alleviating effect of icariin on the LPS-induced inflammatory response and apoptosis in cardiomyocytes. This effect may be mediated by inhibition of the ROS-dependent $J N K / N F-\kappa B$ pathways. These results provide evidence for the potential application of icariin in the treatment of inflammatory injury in cardiovascular diseases.

\section{Acknowledgements}

This study was supported by the National Natural Science Foundation of China (grant nos. 81270303 and 81300070) and the Fundamental Research Funds for the Central Universities of China (grant no. 2012302020212).

\section{References}

1. Coggins $M$ and Rosenzweig A: The fire within: cardiac inflammatory signaling in health and disease. Circ Res 110: 116-125, 2012.

2. Hohensinner PJ, Niessner A, Huber K, Weyand CM and WojtaJ: Inflammation and cardiac outcome. Curr Opin Infect Dis 24: 259-264, 2011.

3. Atefi G, Zetoune FS, Herron TJ, et al: Complement dependency of cardiomyocyte release of mediators during sepsis. FASEB J 25: 2500-2508, 2011.

4. Avlas O, Fallach R, Shainberg A, Porat E and Hochhauser E: Toll-like receptor 4 stimulation initiates an inflammatory response that decreases cardiomyocyte contractility. Antioxid Redox Signal 15: 1895-1909, 2011.

5. Fallach R, Shainberg A, Avlas O, et al: Cardiomyocyte Toll-like receptor 4 is involved in heart dysfunction following septic shock or myocardial ischemia. J Mol Cell Cardiol 48: 1236-1244, 2010.

6. Hua F, Ha T, Ma J, et al: Protection against myocardial ischemia/reperfusion injury in TLR4-deficient mice is mediated through a phosphoinositide 3-kinase-dependent mechanism. J Immunol 178: 7317-7324, 2007.

7. Satoh M, Nakamura M, Akatsu T, Shimoda Y, Segawa I and Hiramori K: Toll-like receptor 4 is expressed with enteroviral replication in myocardium from patients with dilated cardiomyopathy. Lab Invest 84: 173-181, 2004.

8. Frantz S, Kobzik L, Kim YD, et al: Toll4 (TLR4) expression in cardiac myocytes in normal and failing myocardium. J Clin Invest 104: 271-280, 1999.

9. Ha T, Li Y, Hua F, et al: Reduced cardiac hypertrophy in toll-like receptor 4-deficient mice following pressure overload. Cardiovasc Res 68: 224-234, 2005.
10. Xu CQ, Liu BJ, Wu JF, et al: Icariin attenuates LPS-induced acute inflammatory responses: involvement of PI3K/Akt and NF-kappaB signaling pathway. Eur J Pharmacol 642: 146-153, 2010.

11. Wu J, Zhou J, Chen X, et al: Attenuation of LPS-induced inflammation by ICT, a derivate of icariin, via inhibition of the CD14/TLR4 signaling pathway in human monocytes. Int Immunopharmacol 12: 74-79, 2012.

12. Li WW, Gao XM, Wang XM, Guo H and Zhang BL: Icariin inhibits hydrogen peroxide-induced toxicity through inhibition of phosphorylation of JNK/p38 MAPK and p53 activity. Mutat Res 708: 1-10, 2011.

13. Song YH, Cai H, Gu N, Qian CF, Cao SP and Zhao ZM: Icariin attenuates cardiac remodelling through down-regulating myocardial apoptosis and matrix metalloproteinase activity in rats with congestive heart failure. J Pharm Pharmacol 63: 541-549, 2011.

14. Chung BH, Kim JD, Kim CK, et al: Icariin stimulates angiogenesis by activating the MEK/ERK- and PI3K/Akt/eNOS-dependent signal pathways in human endothelial cells. Biochem Biophys Res Commun 376: 404-408, 2008.

15. Zhou H, Bian ZY, Zong J, et al: Stem cell antigen 1 protects against cardiac hypertrophy and fibrosis after pressure overload. Hypertension 60: 802-809, 2012

16. Zhou H, Yang HX, Yuan Y, et al: Paeoniflorin attenuates pressure overload-induced cardiac remodeling via inhibition of TGF $\beta /$ Smads and NF- $\kappa$ B pathways. J Mol Histol 44: 357-367, 2013.

17. Zhou H, Shen DF, Bian ZY, et al: Activating transcription factor 3 deficiency promotes cardiac hypertrophy, dysfunction, and fibrosis induced by pressure overload. PLoS One 6: e26744, 2011.

18. Pan LL, Liu XH, Gong QH and Zhu YZ: S-Propargyl-cysteine (SPRC) attenuated lipopolysaccharide-induced inflammatory response in $\mathrm{H} 9 \mathrm{c} 2$ cells involved in a hydrogen sulfide-dependent mechanism. Amino Acids 41: 205-215, 2011.

19. Dong M, Hu N, Hua Y, et al: Chronic Akt activation attenuated lipopolysaccharide-induced cardiac dysfunction via Akt/GSK3 $\beta$-dependent inhibition of apoptosis and ER stress. Biochim Biophys Acta 1832: 848-863, 2013.

20. González A, Ravassa S, Beaumont J, López B and Díez J: New targets to treat the structural remodeling of the myocardium. J Am Coll Cardiol 58: 1833-1843, 2011.

21. Verma SK, Krishnamurthy P, Barefield D, et al: Interleukin-10 treatment attenuates pressure overload-induced hypertrophic remodeling and improves heart function via signal transducers and activators of transcription 3-dependent inhibition of nuclear factor- $\kappa$ B. Circulation 126: 418-429, 2012.

22. Zeng KW, Fu H, Liu GX and Wang XM: Icariin attenuates lipopolysaccharide-induced microglial activation and resultant death of neurons by inhibiting TAK1/IKK/NF-kappaB and JNK/p38 MAPK pathways. Int Immunopharmacol 10: 668-678, 2010.

23. Mann DL: Inflammatory mediators and the failing heart: past, present, and the foreseeable future. Circ Res 91: 988-998, 2002.

24. Wang YK and Huang ZQ: Protective effects of icariin on human umbilical vein endothelial cell injury induced by $\mathrm{H} 2 \mathrm{O} 2$ in vitro. Pharmacol Res 52: 174-182, 2005.

25. Tien YC, Lin JY, Lai CH, et al: Carthamus tinctorius L. prevents LPS-induced TNFalpha signaling activation and cell apoptosis through JNK1/2-NFkappaB pathway inhibition in H9c2 cardiomyoblast cells. J Ethnopharmacol 130: 505-513, 2010.

26. Ceylan-Isik AF, Zhao P, Zhang B, Xiao X, Su G and Ren J: Cardiac overexpression of metallothionein rescues cardiac contractile dysfunction and endoplasmic reticulum stress but not autophagy in sepsis. J Mol Cell Cardiol 48: 367-378, 2010.

27. Jones WK, Brown M, Ren X, He S and McGuinness M: NF-kappaB as an integrator of diverse signaling pathways: the heart of myocardial signaling? Cardiovasc Toxicol 3: 229-254, 2003.

28. Liu Q, Chen Y, Auger-Messier M and Molkentin JD: Interaction between $\mathrm{NF}_{\kappa} \mathrm{B}$ and NFAT coordinates cardiac hypertrophy and pathological remodeling. Circ Res 110: 1077-1086, 2012.

29. Hayden MS and Ghosh S: Shared principles in NF-kappaB signaling. Cell 132: 344-362, 2008. 